\title{
Analysis of STAT Laboratory Turnaround Times Before and After Conversion of the Hospital Information System
}

\author{
Gary R Lowe MEd RRT-NPS RPFT, Yolanda Griffin MEd RRT, \\ and Michael D Hart MSc RN-BC
}

\begin{abstract}
BACKGROUND: Modern electronic health record systems (EHRS) reportedly offer advantages including improved quality, error prevention, cost reduction, and increased efficiency. This project reviewed the impact on specimen turnaround times (TAT) and percent compliance for specimens processed in a STAT laboratory after implementation of an upgraded EHRS. METHODS: Before EHRS implementation, laboratory personnel received instruction and training for specimen processing. One laboratory member per shift received additional training. TAT and percent compliance data sampling occurred 4 times monthly for 13 months post-conversion and were compared with the mean of data collected for 3 months pre-conversion. Percent compliance was gauged using a benchmark of reporting $95 \%$ of all specimens within 7 min from receipt. RESULTS: Control charts were constructed for TAT and percent compliance with control limits set at 2 SD and applied continuously through the data collection period. TAT recovered to pre-conversion levels by the 6th month post-conversion. Percent compliance consistently returned to pre-conversion levels by the 10th month post-conversion. Statistical analyses revealed the TAT were significantly longer for 3 months post-conversion $(P<.001)$ compared with pre-conversion levels. Statistical significance was not observed for subsequent groups. Percent compliance results were significantly lower for 6 months post-conversion $(P<.001)$. Statistical significance was not observed for subsequent groups. CONCLUSIONS: Extensive efforts were made to train and prepare personnel for challenges expected after the EHRS upgrade. Specific causes identified with the upgraded EHRS included multiple issues involving personnel and the EHRS. These data suggest that system and user issues contributed to delays in returning to pre-conversion TAT and percent compliance levels following the upgrade in the EHRS. Key words: electronic health records; electronic medical records; hospital information system; laboratory information system; turnaround times; computerized patient record. [Respir Care 2014;59(8):1275-1280. (C) 2014 Daedalus Enterprises]
\end{abstract}

\section{Introduction}

The Respiratory Care Department at Arkansas Children's Hospital (Little Rock, Arkansas) utilizes various aspects of electronic health record system (EHRS) functionality, including documentation of respiratory care interventions,

\footnotetext{
Mr Lowe and Ms Griffin are affiliated with Respiratory Care Services, Arkansas Children's Hospital, Little Rock, Arkansas. Mr Hart is affiliated with Arkansas Children's Hospital, Little Rock, Arkansas.
}

Mr. Lowe presented a version of this paper at the AARC Congress 2011, held November 5-8, 2011, in Tampa, Florida.

The authors have disclosed no conflicts of interest. reporting laboratory results via the laboratory information system (LIS), and barcode scanning for medication administration into the electronic health record (EHR). However, various limitations existed in the historical EHRS platform that would not allow for development and integration of more intricate enhancements (eg, computerized provider order entry and computer decision support). As a result, there was an organization-wide upgrade to a modernized

Correspondence: Gary R Lowe MEd RRT-NPS RPFT, Respiratory Care, Slot 303, 1 Children's Way, Little Rock, AR 72202-3591. E-mail: lowegr@archildrens.org.

DOI: $10.4187 /$ respcare. 01814 
application to improve EHRS capabilities. This report relates specifically to the difference in turnaround times (TAT) and percent compliance (transmitting results in a 7-min time frame) of laboratory specimens processed in the Respiratory Care Services STAT laboratory before and after conversion to the new EHRS. The purpose of this project was to determine whether TAT and percent compliance would reach pre-conversion baseline levels and the time frame that would be required to achieve these levels.

\section{Methods}

This project was administratively reviewed by the University of Arkansas for Medical Sciences Institutional Review Board, which determined that the project was not human subject research, as it was considered a quality improvement project. A literature review resulted in no specific information detailing the impact on TAT and percent compliance with reporting benchmarks during transition to newer EHRS technology. The STAT laboratory analyzes and reports the results of laboratory specimens utilizing whole blood analysis and is accredited by the College of American Pathologists. The analytes reported include $\mathrm{pH}, \mathrm{P}_{\mathrm{CO}_{2}}, \mathrm{P}_{\mathrm{O}_{2}}$, sodium, chloride, potassium, ionized calcium, glucose, lactate, total hemoglobin, and fractional values for oxyhemoglobin, carboxyhemoglobin, methemoglobin, and reduced hemoglobin. Calculated values are also reported, including $\mathrm{HCO}_{3}{ }^{-}$, base excess, total $\mathrm{CO}_{2}$, hematocrit, and anion gap. A single specimen yields a result for all analytes, but only results that have been ordered are reported in the LIS. The area is staffed with 2 individuals at all times. STAT laboratory personnel have received specialized training to operate, maintain, and troubleshoot all of the equipment associated with a traditional laboratory, including benchtop and point-of-care devices.

The flow of specimen processing both pre-conversion and post-conversion was as follows: (1) Specimens arrive in the STAT laboratory via a pneumatic tube system. (2) Specimens are analyzed. (3) Results are reviewed by laboratory personnel. (4) Results are electronically filed. (5) Results appear immediately in the patient's EHR for review by medical personnel. (6) Results are automatically sent to a dedicated printer at the patient's location, and a hard copy for review is immediately available.

To use more advanced clinical processes within the EHRS, a change in technology was necessary. The new system operates on an entirely different software code base (back end) and has a different user interface (front end). This essentially was the equivalent of changing to a completely new EHRS. The user interface would change from one common to Microsoft DOS environments to a modern graphical user interface common to Microsoft Windows environments, resulting in an entirely different view for users.

\section{QUICK LOOK}

\section{Current knowledge}

Proposed advantages of an electronic health record (EHR) include improved quality, reduced costs, enhanced efficiency, and fewer errors. Implementation of an EHR should positively impact specimen turnaround time, but there is little study in this area.

\section{What this paper contributes to our knowledge}

Despite extensive training efforts, turnaround times were slower following introduction of the EHR. Identification of issues and corrective action required 6 months to return turnaround times to baseline values. The data suggest that during introduction of the EHR, similarity to existing processes, contingency plans, and parallel testing can reduce inefficiency.

Before the conversion, laboratory personnel were required to complete several phases of training. First, a general introduction to the new platform (in modular format) was required, which took $6 \mathrm{~h}$ to complete. Competency was assessed by successful completion of post-module tests. Second, STAT laboratory-specific training sessions allowed utilization of the LIS in a test mode and required $4 \mathrm{~h}$ to complete. Specific functions included ordering, receiving, editing, result processing, canceling, and rejecting specimens in the LIS. One laboratory member from each shift received $5 \mathrm{~h}$ of supplemental training and was a designated superuser for the shift. Superuser training included resolution of potential problems (eg, results not translating to the LIS, results not printing to a dedicated printer, and general troubleshooting) that might occur after the conversion. There were $2 \mathrm{~d}$ of actual mock testing of the system where test patients were entered into the system, and specimens were processed through each area of the hospital (from beginning to end of an in-patient or out-patient visit). The training and specimen processing were observed and monitored by the STAT laboratory supervisor and a representative from the information technology (IT) department. Eleven days before the conversion, laboratory personnel were permitted unlimited access to the test mode and practiced processing mock specimens. Additionally, clinical personnel were trained on use of the system through self-guided interactive training modules. Designated superusers for each patient care area were trained to support clinical users in their initial use of the system once the conversion took place.

During the training sessions, it was noted that one additional step was added with the new EHRS application. This step required the actual specimen acquisition time to be documented in the electronic requisition. Once the con- 


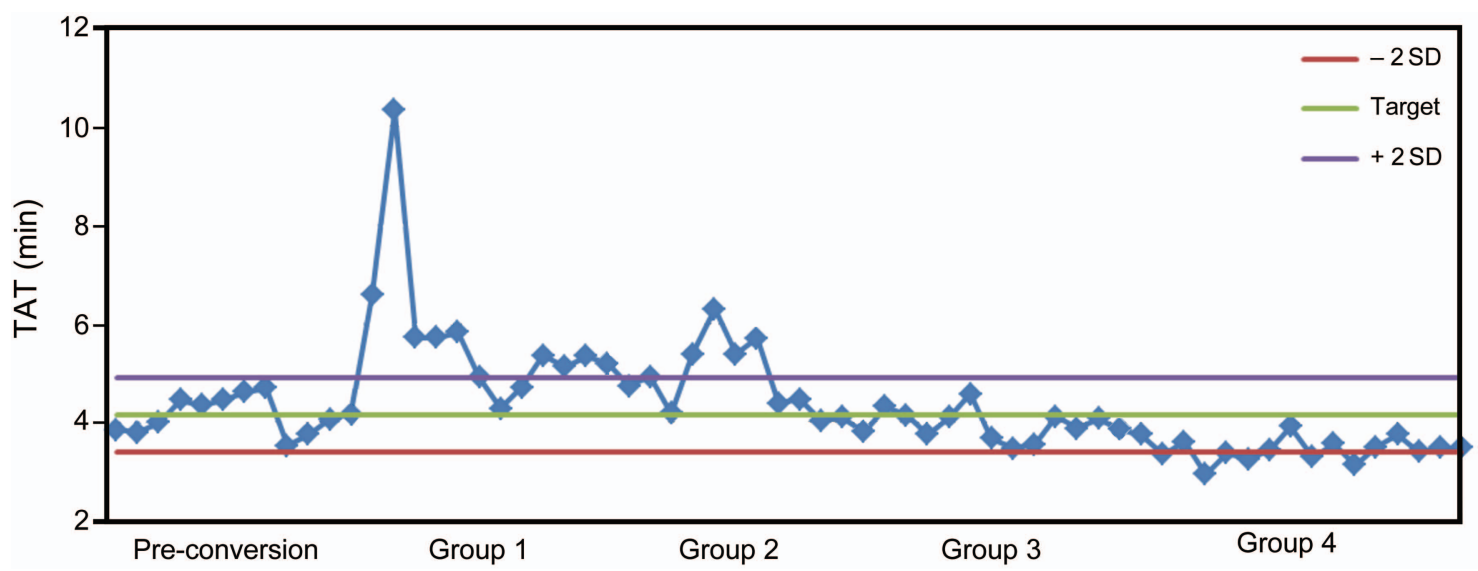

Fig. 1. Control chart of average turnaround times (TAT) of specimens processed during each week of the observation.

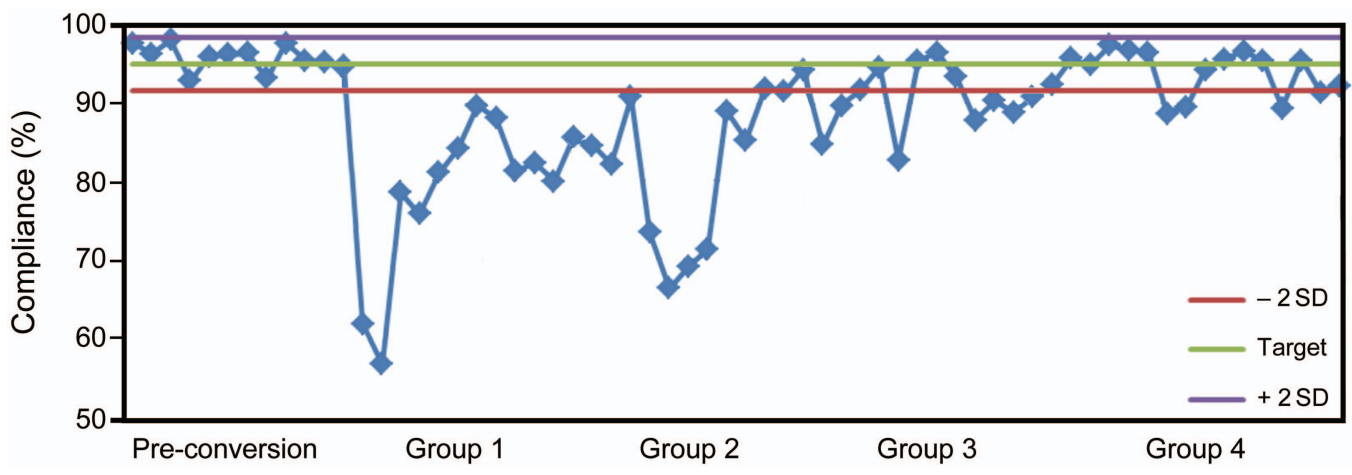

Fig. 2. Control chart of average percent compliance of specimens processed during each week of the observation.

version occurred, delays in electronic result reporting occurred due to the system being taken offline for system resets and software adjustments. Reversion to paper requisition and reporting mechanisms had to be initiated. It soon became clear that the clinical staff and STAT laboratory personnel were challenged by the EHRS changes. This retrospective review compared STAT laboratory TAT and percent compliance before and after conversion to the new EHRS platform. TAT was the average time of all specimens processed in a $24-\mathrm{h}$ time period from receipt in the STAT laboratory to result transmission, and percent compliance was gauged using an internal benchmark of reporting $95 \%$ of all specimens processed within $7 \mathrm{~min}$ from receipt to result transmission. Data were collected monthly for 13 months after the conversion to the upgraded EHRS and compared with the mean of data collected for 3 months pre-conversion. Pre-conversion and post-conversion data sampling was conducted 4 times monthly (every 7-8 d) and represented a sample of specimens processed by all shifts. The post-conversion data points were analyzed on an individual basis and compared with the 3-month pre-conversion average. Additionally, these 4 data points were aggregated to provide a monthly composite and compared with the pre-conversion average.
At the end of each month, the information was conveyed to senior leadership for review. The information was also posted in the STAT laboratory for personnel to review and provide timely feedback on their performance.

\section{Results}

The first step in this analysis was to construct control charts for TAT (Fig. 1) and percent compliance (Fig. 2). For TAT, the pre-conversion mean $\pm \mathrm{SD}$ was $4.15 \pm 0.37$ min. The post-conversion means \pm SD were $5.76 \pm 1.56$ $\min$ for group 1, $4.79 \pm 0.77 \mathrm{~min}$ for group 2, 3.96 \pm 0.32 $\min$ for group 3 , and $3.45 \pm 0.24$ min for group 4 . Control limits were set at $2 \pm 0.74$ min derived from pre-conversion data and applied continuously through the graph to depict outliers following the conversion. TAT remained above the upper control limit for 5 months post-conversion, indicating longer TAT, and recovered to pre-conversion levels by the 6th month post-conversion. They remained at pre-conversion levels for an additional 3 months and then began trending lower, which resulted in TAT that actually improved compared with the pre-conversion levels. For percent compliance, the pre-conversion mean \pm SD 
Table 1. Analysis of Grouped and Overall Post-Conversion Percent Compliance and Turnaround Times Compared With PreConversion Values

\begin{tabular}{|c|c|c|c|c|}
\hline Group & $\begin{array}{c}\% \\
\text { Compliance }\end{array}$ & $P^{*}$ & $\begin{array}{c}\text { Turnaround } \\
\text { Times (min) }\end{array}$ & $P^{*}$ \\
\hline Pre-conversion group & $95.8 \pm 1.67$ & & $4.15 \pm 0.37$ & \\
\hline $\begin{array}{l}\text { Post-conversion group } 1 \\
\quad(0-3 \text { mo })\end{array}$ & $78.7 \pm 10.0$ & $<.001$ & $5.76 \pm 1.56$ & $<.001$ \\
\hline $\begin{array}{l}\text { Post-conversion group } 2 \\
\quad(4-6 \mathrm{mo})\end{array}$ & $82.4 \pm 9.93$ & $<.001$ & $4.79 \pm 0.77$ & .29 \\
\hline $\begin{array}{l}\text { Post-conversion group } 3 \\
\quad(7-9 \text { mo })\end{array}$ & $90.5 \pm 4.19$ & .29 & $3.96 \pm 0.32$ & .98 \\
\hline $\begin{array}{l}\text { Post-conversion group } 4 \\
\quad(10-13 \mathrm{mo})\end{array}$ & $93.8 \pm 2.92$ & .94 & $3.45 \pm 0.24$ & .16 \\
\hline $\begin{array}{l}\text { Overall post-conversion } \\
\quad(0-13 \mathrm{mo})\end{array}$ & $86.9 \pm 9.38$ & .002 & $4.41 \pm 1.22$ & .46 \\
\hline
\end{tabular}

was $95.8 \pm 1.67 \%$. The post-conversion means \pm SD were $78.7 \pm 10.0 \%$ for group $1,82.4 \pm 9.93 \%$ for group $2,90.5 \pm 4.19 \%$ for group 3 , and $93.8 \pm 2.92 \%$ for group 4. Control limits were set at $2 \pm 3.34 \%$ derived from pre-conversion data and applied continuously through the graph to depict outliers following the conversion. Unlike TAT, the percent compliance took longer to recover but consistently returned to pre-conversion levels by the 10th month post-conversion.

The second step included data analysis utilizing statistical software (SPSS 16.0, SPSS, Chicago, Illinois). Results are presented as mean \pm SD. Pre-conversion data consisted of 3 months of data (one value per week for a total of 12 values) that were analyzed to determine the mean TAT and percent compliance in $12.1 \%(2,347 / 19,389)$ of total specimens processed. The mean values were used as the baseline benchmark for comparisons with post-conversion data. Post-conversion data were grouped in 3-month increments through month 9: group 1,0-3 months postconversion; group 2, 4-6 months post-conversion; and group 3, 7-9 months post-conversion. Group 4 had 4 months of data (10-13 mo post-conversion). Post-conversion data were collected in a similar manner (one value per week) in $11.6 \%(8,619 / 73,770)$ of total specimens processed. A oneway analysis of variance with the Tukey honest significant difference test was used for comparisons made between the pre-conversion data and grouped post-conversion data, with $P \leq .05$ considered significant. Results are shown in Table 1 . TATS were significantly higher in group 1 postconversion $(P<.001)$ compared with pre-conversion levels. Statistical significance was not observed for subsequent groups. Percent compliance was significantly lower in groups 1 and $2(P<.001)$ post-conversion compared with pre-conversion levels, and statistical significance was not observed for subsequent groups. However, for the entire observation period, when post-conversion percent compliance was compared with pre-conversion data, the mean was significantly lower $(P=.002)$.

TAT post-conversion levels, according to the control charts and statistical analyses, returned to pre-conversion levels within 3-6 months. Percent compliance levels returned to pre-conversion levels between 6 and 9 months. During the observation period, there were no negative clinical effects or adverse events noted with the delays in result reporting.

\section{Discussion}

The American Recovery and Reinvestment Act of 2009 included provisions that in aggregate comprised the Health Information Technology for Economic and Clinical Health (HITECH) Act. ${ }^{1}$ The HITECH Act is focused on improving health-care quality, safety, and efficiency through promotion of health information technology, notably EHR, and through greater electronic exchange of health information. ${ }^{1,2}$ The legislation ties payments specifically to the achievement of advances in health-care processes and outcomes. ${ }^{3}$ Adoption of the modernized EHRS has been extremely slow despite the potential financial incentives in utilizing systems that fulfill the definition of meaningful use as outlined in the HITECH Act. ${ }^{4}$ In a recent survey, Jha et $\mathrm{al}^{5}$ found that $>75 \%$ of hospitals reported adoption of electronic laboratory and radiologic reporting systems. Our institution adopted an electronic laboratory reporting system in the early 1990s. The transition process to the new application showed a significant increase in TAT and decrease in percent compliance initially; however, laboratory personnel had familiarity with utilizing an LIS before the conversion. This suggests the possibility that the process change to the new EHRS platform initially impacted their ability to process specimens as efficiently as they did before the conversion.

The results indicate that the STAT laboratory was within the defined internal benchmark of average TAT for specimens processed in $<7$ min by the 2 nd month post-conversion; however, the purpose of this observation was to determine whether and when the TAT returned to the levels noted pre-conversion. There are no national guidelines regarding defined TAT. According to the College of American Pathologists Laboratory General Checklist, "The laboratory has defined turnaround times (ie, the interval between specimen receipt by laboratory personnel and results reporting) for each of its tests," ${ }^{6}$ but no specific TAT are indicated. These ranges are determined by the laboratory and approved by the STAT laboratory medical director. Also, one would think that when TAT averages returned to $<7$ min, compliance would also recover. However, this was not the case. For example, if the STAT laboratory 
processed 100 samples in $24 \mathrm{~h}, 95$ of the samples would have to have a TAT of $<7$ min to attain the $95 \%$ compliance target. If 8 samples had a TAT of $>7 \mathrm{~min}$, then compliance would be $92 \%$ for that day, even though the overall average TAT could be $<7 \mathrm{~min}$.

The additional step identified during the testing phase (documenting the specimen acquisition time) negatively influenced the TAT and percent compliance to a greater degree than originally anticipated. This step required ordering personnel to log into the system and document the time the specimen was collected. Orders could be placed up to $12 \mathrm{~h}$ in advance; however, the collection time had to be entered when the specimen was obtained to complete the order. If this step was not performed, the order could not be retrieved by laboratory personnel to acquire the accession number. To rectify this omission, laboratory personnel had to contact and request ordering personnel to log into the system and document the time the specimen was collected. We speculate that this potentially led to delays in specimen processing. No methodology was devised to separate these results from the overall TAT and percent compliance results to identify the scope of this issue. This additional step proved to be a significant barrier postconversion, and it is highly recommended that others undergoing a similar transition should focus on any process changes and devise ways to isolate and analyze the impact of any differences noted during the pre-conversion testing and training. It is suspected that the impact of this step was gradually eliminated as clinical staff adapted to the requirements of the new system.

In an attempt to offset anticipated problems (EHRS being taken offline for system resets and software adjustments), procedural steps included a mixture of paper and electronic steps. This method attempted to provide a means for ensuring that required information was available to STAT laboratory personnel in the event the information was missing from the electronic documentation. For example, a paper requisition had to be sent with the specimen to ensure that the required elements for proper documentation were available for entry into the system. These additional requirements may have amplified delays with specimen result processing and negatively impacted TAT and percent compliance during the early post-conversion phase. Again, this is speculative since the data were analyzed in aggregate, and no data were eliminated from the data pool.

Are there other ways to potentially improve the outcomes of a transition to a new EHRS/LIS that will minimize slowdowns and disruptions in the reporting of laboratory results? A recent Institute of Medicine report stated, "Extensive training must be done for the specific product and the specific organizational setting. It is customary for organizations to set expectations for training that require documentation of learning modules and demonstrated competency."7 Before the transition, hospital personnel partic- ipated in training processes to prepare for the conversion as described previously. This is one area identified that may not have been utilized to the fullest potential. We were unable to audit the actual time spent by individual users in the test mode. Our experience showed that the training process should have included an audit of time spent by laboratory personnel in the test mode and in processing mock specimens and should have required that the staff document time spent in training. Buntin et $\mathrm{al}^{8}$ states that 'the 'human element' is critical to health IT implementation, and this highlights the importance of strong leadership and staff 'buy in' if systems are to successfully manage and see benefit from health information technology." Despite the pre-conversion training requirements and monitoring by the supervisor and IT staff, the clinical staff and laboratory personnel initially struggled with the changes after the conversion. The adaptation to the new system took several months to return to pre-conversion performance levels.

Another aspect to consider is whether implementation should be a comprehensive versus a gradual conversion. According to the Institute of Medicine, "an organization selects one of two approaches to implementing the technology: either a big bang strategy (ie, the technology is implemented for use throughout the entire organization at the same time) or an incremental approach (ie, the technology is first deployed for use on a small scale within the organization and then, as operating experience is acquired, it is deployed to other parts of the organization in a gradual staged manner). Both approaches can be successful."7 The incremental approach was not an option due to the integration of the new application. This conversion used the big bang strategy, and once the conversion started, it could not be reversed. Use of paper downtime requisitions occurred during several points after the initial conversion as a result of taking the system offline for system resets, software fixes, and upgrades. These negatively impacted TAT and percent compliance. With the benefit of hindsight, we would have set more realistic expectations about the amount of time needed for the STAT laboratory's performance to return to pre-conversion levels. Although there are few reports that delineate a certain length of time for learning a new system (eg, $\sim 6$ months), for better understanding of the system's effects, certain aspects may need to be assessed longitudinally. ${ }^{9,10}$ The recovery time for the TAT returning to and staying at baseline took $\sim 5$ months. However, percent compliance took $\sim 10$ months to recover to baseline. These may be realistic expectations when a complex implementation of this magnitude occurs.

The observed increase in TAT and percent compliance had multiple causes. There were delays with specimen processing at various points between users ordering, collecting, documenting, and sending specimens and laboratory personnel receiving, analyzing, result processing, and 
filing the results. Specific system causes included personnel issues (eg, realistic training to simulate the EHRS in preparation for the conversion, getting acclimated to the differences within the EHRS, and callbacks for missing or incomplete information) and system issues (eg, technical problems, software upgrades, and system downtime) that forced staff to go to downtime processes and revert to paper forms for processing specimens. Although there was an initial increase in TAT and percent compliance, it turned out to be a reasonable trade-off to improve performance and quality in more global ways. The upgraded EHRS enabled us to utilize more advanced functionality that potentially impacts every patient throughout our system.

There are several limitations that should be discussed. First, our results depend on our own internal benchmark, which has been established over time and with multidisciplinary input and review. We are confident that these benchmarks accurately reflect the realities of our setting; however, their generalizability will require applying benchmarks established by others at their own institution. Second, these results are specific to the EHRS/LIS we utilize and are not generalizable to those obtained with other EHRS/LIS systems. Third, this is a dynamic process, and as interventions are made, a review of the applicability of this model should be performed and the necessary modifications made to set reasonable goals that are realistically attainable by the staff.

\section{Conclusions}

Extensive efforts were made to ensure that laboratory and clinical personnel were adequately trained to handle problems anticipated post-conversion. TAT recovered to the pre-conversion benchmark within 3-6 months. Attainment of compliance with the benchmark of processing $95 \%$ of specimens within 7 min took $6-9$ months to reach. Despite training and actual experience, it took a significant amount of time to assimilate the new system. Based on our experience, when transitioning to a new EHRS/LIS, the following recommendations may offset a lengthy return to pre-conversion levels of TAT and percent compliance:
(1) minimize the differences between the old system and the new system; (2) when possible, conduct parallel testing using real patient data and real patient volumes to identify the impact of the new processes under actual working conditions; (3) develop well defined contingency processes that are fast and easy to implement when the new system fails to work properly; and (4) train clinicians as to when and how to implement contingency processes and obtain interdepartmental support. These interventions may offset or minimize the impact on TAT and percent compliance of laboratory specimen processing after an EHRS conversion of this magnitude.

\section{REFERENCES}

1. Henricks, WH. "Meaningful use" of electronic health records and its relevance to laboratories and pathologists. J Pathol Inform 2011;2:7.

2. Blumenthal D. Stimulating the adoption of health information technology (perspective). N Engl J Med 2009;360(15):1477-1479.

3. Blumenthal D, Tavenner M. The "meaningful use" regulation for electronic health records (perspective). N Engl J Med 2010;363(6): 501-504.

4. Abramson EL, Malhotra S, Fischer K, Edwards A, Pfoh ER, Osorio $\mathrm{SN}$, et al. Transitioning between electronic health records: effects on ambulatory prescribing safety. J Gen Intern Med 2011;26(8):868874.

5. Jha AK, DesRoches CM, Campbell EG, Donelan K, Rao SR, Ferris TG, et al. Use of electronic health records in U.S. hospitals. N Engl J Med 2009;360(16):1628-1638.

6. Commission on Laboratory Accreditation. Laboratory general accreditation checklist. Northfield, Illinois: College of American Pathologists; 2011. http://www.cap.org/apps/docs/education/ OnlineCourseContent/2011/LAP/Resources/Checklists/LabGen.pdf. Accessed June 25, 2014.

7. Institute of Medicine. Health IT and patient safety: building safer systems for better care. Washington, DC: The National Academies Press; 2011. http://www.iom.edu/Reports/2011/Health-IT-andPatient-Safety-Building-Safer-Systems-for-Better-Care.aspx. Accessed June 19, 2014.

8. Buntin MB, Burke MF, Hoaglin MC, Blumenthal D. The benefits of health information technology: a review of the recent literature shows predominately positive results. Health Affairs 2011;30(3):464-471.

9. Nahm ES, Vaydia V, Ho D, Scharf B, Seagull J. Outcomes assessment of clinical information system implementation: a practical guide. Nursing Outlook 2007;55(6):282-288.

10. Blignaut PJ, McDonald T, Tolmie CJ. Predicting the learning and consultation time in a computerized primary healthcare clinic. Comput Nurs 2001;19(3):130-136. 